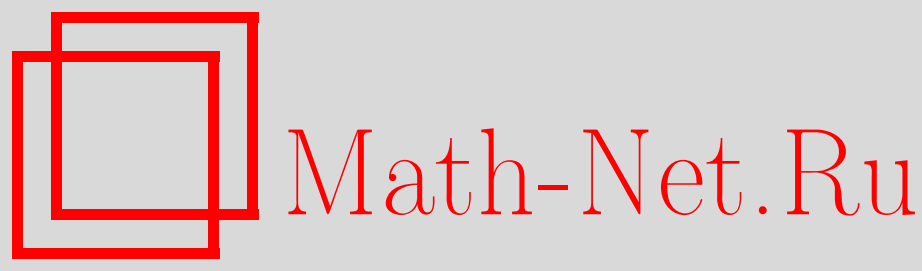

Дан Тунг Нгун, Н. М. Плакида, Статическая зарядовая восприимчивость в $t-J-V$ модели, TMФ, 2018, том 194, номер 1, 151-167

DOI: https://doi.org/10.4213/tmf9393

Использование Общероссийского математического портала Math-Net.Ru подразумевает, что вы прочитали и согласны с пользовательским соглашением http://www . mathnet.ru/rus/agreement

Параметры загрузки:

IP : 3.81 .55 .215

26 апреля 2023 г., $07: 05: 31$

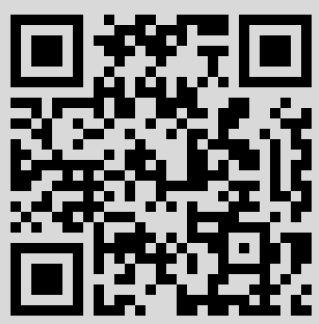




\title{
СТАТИЧЕСКАЯ ЗАРЯДОВАЯ ВОСПРИИМЧИВОСТЬ В $t-J-V$ МОДЕЛИ
}

\begin{abstract}
Рассматривается статическая зарядовая восприимчивость и корреляционная функция плотности зарядов в двумерной $t-J-V$ модели на основе метода уравнений движения для функции релаксации от операторов Хаббарда. Получена зависимость восприимчивости и корреляционной функции от концентрации дырок и температуры. Показано, что при достаточно сильном межузельном кулоновском взаимодействии в системе возможно появление волн зарядовой плотности.
\end{abstract}

Ключевые слова: сильные электронные корреляции, зарядовая восприимчивость, высокотемпературная сверхпроводимость, $t-J-V$ модель.

DOI: https://doi.org/10.4213/tmf9393

\section{1. ВВЕДЕНИЕ}

Двухвременные температурные функции Грина $(Ф Г)$, введенные в статистическую физику Боголюбовым и Тябликовым [1], [2], оказались весьма удобным инструентом изучения спектра возбуждений и фазовых переходов в многочастичных системах [3], [4]. Особенно эффективным в построении цепочки уравнений оказался метод проектирования [5], [6]. Используя этот метод, удается получить точное представление для ФГ с массовым или поляризационным оператором, который далее вычисляется самосогласованным образом через полные ФГ. Таким образом, можно рассматривать системы с сильным взаимодействием, не вводя нулевые ФГ, как это делается в температурной диаграммной технике. Как было показано нами в работе [7], этот метод позволяет вычислить спектр спиновых волн и намагниченность в ферромагнитной модели Гейзенберга в полном соответствии с результатами температурной диаграммной техники в упорядоченной фазе и рассмотреть спектр спиновых флуктуаций в парамагнитной области. С помощью этого метода нами были также вычислены спектры антиферромагнитных спиновых возбуждений для высокотемпературных сверхпроводников в рамках $t$ - $J$ модели как в нормальной [8], так и в сверхпроводящей фазах [9], а также оптическая проводимость [10].

* Объединенный институт ядерных исследований, Дубна, Московская обл., Россия. E-mail: plakida@theor.jinr.ru 
В настоящей работе мы воспользуемся методом проектирования для ФГ для исследования волн зарядовой плотности (ВЗП). В последнее время это явление наблюдалось в многочисленных экспериментах в медно-оксидных соединениях при небольшой концентрации носителей заряда. ВЗП были обнаружены в соединениях YBCO (см. [11]-[14] и цитированную там литературу), Bi-2201 [15], Bi-2212 [16]-[18], Hg-1201 [19], [20]. ВЗП во всех соединениях имеют близкие характеристики: они возникают в плоскости $\mathrm{CuO}_{2}$ при температурах $T_{\mathrm{CDW}}$ меньше или порядка температуры появления псевдощелевого состояния $T^{*}$, но выше температуры свехпроводящего перехода $T_{\mathrm{c}}$, определяются несоразмерным волновым вектором $\left(Q_{x}, Q_{y}\right)=0.2-0.3$ (в единицах обратной решетки), имеют небольшую корреляционную длину порядка 5-8 постоянных решетки. Модуляция заряда происходит на узлах кислородной подрешетки и имеет $d$-симметрию, т. е. разный знак на узлах кислорода в одной элементарной ячейке.

При теоретическом описании ВЗП в купратах рассматривались как системы с сильной корреляцией, так и системы в приближении ферми-жидкости (см. [21] и цитированную там литературу). В первом случае обычно используется $t$ - $J$ модель с учетом межузельного кулоновского отталкивания, так называемая $t-J-V$ модель. В частности, в работах [22]-[24] в рамках этой модели образование псевдощели в нормальном состоянии купратов объясняется возникновением ВЗП при учете кулоновского и обменного антиферромагнитного взаимодействий. В работе [25] получены аналитические выражения для зарядовой восприимчивости и диэлектрической проницаемости для модели экранированного кулоновского взаимодействия электронов в купратах. В работе [26] анализируется связь ВЗП со смягчением продольной фононной моды в купратах. В работе [27] предложена формула для расчета температурной зависимости лондоновской глубины проникновения магнитного поля в режиме сосуществования ВЗП и сверхпроводимости. Аналитическое выражение для динамической зарядовой восприимчивости было получено в работе [28] при выходе за рамки приближения случайных фаз. Для анализа ВЗП использовались также численные методы, например метод Монте-Карло в работе [29]. Детальное исследование электрон-дырочной неустойчивости в модели ферми-жидкости к образованию ВЗП на узлах кислорода в медно-оксидной плоскости было проведено в работе [21].

В настоящей работе мы вычисляем динамическую зарядовую восприимчивость в рамках $t-J-V$ модели для дырок, пользуясь методом функции релаксации [30]-[32] для ФГ от операторов плотности заряда. Ранее этот подход был использован в рамках стандартной $t$ - $J$ модели в работе [33]. Более подробно мы рассмотрим статическую зарядовую восприимчивость в этой модели. Появление ВЗП характеризуется возникновением расходимости в статической зарядовой восприимчивости при определенных параметрах модели и концентрации дырок. При этом спектр дырочных возбуждений вычисляется также в рамках $t-J-V$ модели в методе проектирования для одночастичных ФГ аналогично работе [34] для $t-J$ модели.

В разделе 2 формулируется модель и вычисляются динамическая и статическая зарядовые восприимчивости. В разделе 3 приводятся результаты для статической зарядовой восприимчивости и анализируются условия появления ВЗП. В приложениях А, Б приводятся детали вычислений. 


\section{2. МОДЕЛЬ И ЗАРЯДОВАЯ ВОСПРИИМЧИВОСТЬ}

2.1. Модель. Рассмотрим систему электронов с сильной кулоновской корреляцией. При небольшой концентрации дырок в качестве основного состояния удобно выбрать однократно заполненную электронную подзону Хаббарда, описывая дырки в рамках $t-J-V$ модели. В этой модели дополнительно к обычным $t$ - и $J$-членам (см., например, [34]) учитывается межузельное кулоновское отталкивание дырок $V$ в решетке. Для описания сильно коррелированных систем необходимо использовать спроектированные операторы электронов (дырок), которые удобно представить в виде операторов Хаббарда [35], [36]. В этих операторах модель представляется в виде

$$
\begin{aligned}
H=H_{t}+H_{J}+H_{c}=\sum_{i \neq j, \sigma} & t_{i j} X_{i}^{\sigma 0} X_{j}^{0 \sigma}-\mu \sum_{i, \sigma} X_{i}^{\sigma \sigma}+ \\
& +\frac{1}{4} \sum_{i \neq j, \sigma} J_{i j}\left(X_{i}^{\sigma \bar{\sigma}} X_{j}^{\bar{\sigma} \sigma}-X_{i}^{\sigma \sigma} X_{j}^{\bar{\sigma} \bar{\sigma}}\right)+\frac{1}{2} \sum_{i \neq j} V_{i j} N_{i} N_{j},
\end{aligned}
$$

где операторы Хаббарда $X_{i}^{\alpha \beta}=|i \alpha\rangle\langle i \beta|$ описывают переходы из состояния $|i, \beta\rangle$ в состояние $|i, \alpha\rangle$ на узле решетки $i$ для трех возможных состояний дырки: незаполненное состояние $(\alpha, \beta=0)$ и два однократно заполненных состояния $(\alpha, \beta=\sigma)$ для дырки со спином $\sigma / 2, \sigma= \pm 1(\bar{\sigma}=-\sigma)$. В гамильтониане (1) мы ввели параметры перескока $t_{i j}=t \delta_{j, i+\mathbf{a}_{1}}+t^{\prime} \delta_{j, i+\mathbf{a}_{2}}+t^{\prime \prime} \delta_{j, i+\mathbf{a}_{3}}$, где $t, t^{\prime}, t^{\prime \prime}$ - параметры перескока между первыми $\mathbf{a}_{1}= \pm a_{x}, \pm a_{y}$, вторыми $\mathbf{a}_{2}= \pm\left(a_{x} \pm a_{y}\right)$ и третьими $\mathbf{a}_{3}= \pm 2 a_{x}, \pm 2 a_{y}$ соседями ( $a_{x}=a_{y}$ - постоянные решетки). Обменное взаимодействие $J_{i j}=J \delta_{j, i+\mathbf{a}_{1}}$ учитывается только для ближайших соседей. Кулоновское взаимодействие для дырок на решетке между первыми и вторыми соседями задается в виде $V_{i j}=V_{1} \delta_{j, i+\mathbf{a}_{1}}+V_{2} \delta_{j, i+\mathbf{a}_{2}}$. При этом для фурье-компонент параметров взаимодействия получаем выражения

$$
\begin{aligned}
t(\mathbf{q}) & =4 t \gamma(\mathbf{q})+4 t^{\prime} \gamma^{\prime}(\mathbf{q})+4 t^{\prime \prime} \gamma^{\prime \prime}(\mathbf{q}), \\
V(\mathbf{q}) & =4 V_{1} \gamma(\mathbf{q})+4 V_{2} \gamma^{\prime}(\mathbf{q}), \\
J(\mathbf{q}) & =4 J \gamma(\mathbf{q}),
\end{aligned}
$$

где зависимость от $\mathbf{q}$ в единицах обратной решетки определяется функциями $\gamma(\mathbf{q})=$ $(1 / 2)\left(\cos q_{x}+\cos q_{y}\right), \gamma^{\prime}(\mathbf{q})=\cos q_{x} \cos q_{y}$ и $\gamma^{\prime \prime}(\mathbf{q})=(1 / 2)\left(\cos 2 q_{x}+\cos 2 q_{y}\right)$.

Операторы числа частиц и спина дырок в представлении операторов Хаббарда имеют вид

$$
\begin{gathered}
N_{i}=\sum_{\sigma} X_{i}^{\sigma \sigma}, \\
S_{i}^{\sigma}=X_{i}^{\sigma \bar{\sigma}}, \quad S_{i}^{z}=\frac{\sigma}{2}\left[X_{i}^{\sigma \sigma}-X_{i}^{\bar{\sigma} \bar{\sigma}}\right] .
\end{gathered}
$$

Химический потенциал $\mu$ в гамильтониане (1) находится из уравнения для среднего числа дырок $n$ :

$$
n=\left\langle N_{i}\right\rangle,
$$

где $\langle\cdot\rangle$ - статистическое среднее с гамильтонианом (1). 
Операторы Хаббарда удовлетворяют условию полноты

$$
X_{i}^{00}+X_{i}^{\sigma \sigma}+X_{i}^{\bar{\sigma} \bar{\sigma}}=1
$$

согласно которому на узле решетки дырка может находиться лишь в одном из квантовых состояний $|\alpha\rangle$, что позволяет точно учесть отсутствие двухкратно заполненных квантовых состояний на каждом узле решетки. Операторы Хаббарда подчиняются правилам умножения $X_{i}^{\alpha \beta} X_{i}^{\gamma \delta}=\delta_{\beta \gamma} X_{i}^{\alpha \delta}$, которые определяют для них коммутационные соотношения

$$
\left[X_{i}^{\alpha \beta} X_{j}^{\gamma \delta}\right]_{ \pm}=\delta_{i j}\left(\delta_{\beta \gamma} X_{i}^{\alpha \delta} \pm \delta_{\delta \alpha} X_{i}^{\gamma \beta}\right),
$$

где верхний знак относится к операторам ферми-типа, $X_{i}^{\sigma 0}$, а нижний знак - к операторам бозе-типа, как операторы числа частиц (5) и спина (6).

2.2. Динамическая зарядовая восприимчивость. Динамическая зарядовая восприимчивость $\chi_{\mathbf{q}}(\omega)$ определяется фурье-компонентой запаздывающей ФГ [3]

$$
\chi_{\mathbf{q}}(\omega)=-\left\langle\left\langle N_{\mathbf{q}} \mid N_{-\mathbf{q}}\right\rangle\right\rangle_{\omega}=i \int_{0}^{\infty} d t e^{i \omega t}\left\langle\left[N_{\mathbf{q}}(t), N_{-\mathbf{q}}\right]\right\rangle,
$$

где фурье-компонента оператора флуктуаций плотности заряда

$$
N_{\mathbf{q}}=\frac{1}{\sqrt{N}} \sum_{i} N_{i} e^{-i \mathbf{q} \mathbf{r}_{i}}
$$

При вычислении ФГ от коллективных переменных, как плотность заряда (11), удобно использовать метод функции релаксации [30]-[32]. Определим функцию релаксации в виде

$$
\Phi_{\mathbf{q}}(\omega)=\left(\left(N_{\mathbf{q}} \mid N_{-\mathbf{q}}\right)\right)_{\omega}=-i \int_{0}^{\infty} d t e^{i \omega t}\left(N_{\mathbf{q}}(t), N_{-\mathbf{q}}\right),
$$

где введено скалярное произведение Кубо-Мори

$$
(A(t), B)=\int_{0}^{\beta} d \lambda\langle A(t-i \lambda) B\rangle, \quad \beta=\frac{1}{k_{\mathrm{B}} T} .
$$

Для ФГ, функции релаксации и корреляционных функций выполняются следующие соотношения:

$$
\begin{aligned}
((i \dot{A} \mid B))_{\omega} & =((A \mid-i \dot{B}))_{\omega}=\langle\langle A \mid B\rangle\rangle_{\omega}, \\
(i \dot{A} B) & =(A,-i \dot{B})=\langle[A, B]\rangle, \\
(A, B) & =-\langle\langle A \mid B\rangle\rangle_{\omega=0},
\end{aligned}
$$

где $i \dot{A}=i d A / d t=[A, H]$. Записывая уравнение для $i d \Phi_{\mathbf{q}}(t) / d t$ и используя эти соотношения, находим связь между динамической зарядовой восприимчивостью $\chi_{\mathbf{q}}(\omega)$ и функцией релаксации $\Phi_{\mathbf{q}}(\omega)$ :

$$
\chi_{\mathbf{q}}(\omega)=\chi_{\mathbf{q}}-\omega \Phi_{\mathbf{q}}(\omega),
$$

где $\chi_{\mathbf{q}}=\chi_{\mathbf{q}}(0)=\left(N_{\mathbf{q}}, N_{-\mathbf{q}}\right)-$ статическая восприимчивость. 
Для вычисления функции релаксации $\Phi_{\mathbf{q}}(\omega)$ воспользуемся методом проектирования [5], [6], составляя уравнения для функции $\Phi_{\mathbf{q}}\left(t-t^{\prime}\right)$ при последовательном дифференцировании ее по двум временам, $t$ и $t^{\prime}$. При этом получаем следующую систему уравнений для фурье-компонент функции релаксации:

$$
\begin{aligned}
\omega\left(\left(N_{\mathbf{q}} \mid N_{-\mathbf{q}}\right)\right)_{\omega} & =\chi_{\mathbf{q}}+\left(\left(i \dot{N}_{\mathbf{q}} \mid N_{-\mathbf{q}}\right)\right)_{\omega}, \\
\omega\left(\left(i \dot{N}_{\mathbf{q}} \mid N_{-\mathbf{q}}\right)\right)_{\omega} & =\left(\left(i \dot{N}_{\mathbf{q}} \mid-i \dot{N}_{-\mathbf{q}}\right)\right)_{\omega},
\end{aligned}
$$

в последнем уравнении согласно (15) неоднородный член равен нулю, $\left(i \dot{N}_{\mathbf{q}}, N_{-\mathbf{q}}\right)=$ $\left\langle\left[N_{\mathbf{q}}, N_{-\mathbf{q}}\right]\right\rangle=0$. Решение этой системы уравнений может быть записано в виде

$$
\Phi_{\mathbf{q}}(\omega)=\Phi_{\mathbf{q}}^{0}(\omega)+\Phi_{\mathbf{q}}^{0}(\omega) T_{\mathbf{q}}(\omega) \Phi_{\mathbf{q}}^{0}(\omega), \quad \Phi_{\mathbf{q}}^{0}(\omega)=\frac{\chi_{\mathbf{q}}}{\omega}
$$

где мы ввели матрицу рассеяния первого порядка как функцию релаксации токов $j_{\mathbf{q}}=\dot{N}_{\mathbf{q}}=-i\left[N_{\mathbf{q}}, H\right]$ :

$$
T_{\mathbf{q}}(\omega)=\frac{1}{\chi_{\mathbf{q}}^{2}}\left(\left(j_{\mathbf{q}} \mid j_{-\mathbf{q}}\right)\right)_{\omega} .
$$

Чтобы учесть только неприводимую часть временно́й зависимости в матрице рассеяния $(20)$, определим функцию памяти $M_{\mathbf{q}}(\omega)=\left(\left(j_{\mathbf{q}} \mid j_{-\mathbf{q}}\right)\right)_{\omega}^{(\mathrm{irr})}$ согласно уравнению

$$
T_{\mathbf{q}}(\omega)=\frac{M_{\mathbf{q}}(\omega)}{\chi_{\mathbf{q}}^{2}}+\frac{M_{\mathbf{q}}(\omega)}{\chi_{\mathbf{q}}^{2}} \Phi_{\mathbf{q}}^{0}(\omega) T_{\mathbf{q}}(\omega),
$$

которая не содержит частей, связанных “нулевой” функцией релаксации $\Phi_{\mathbf{q}}^{0}(\omega)$. Введение неприводимой части временно́й зависимости для функции релаксации токов эквивалентно введению спроектированного оператора Лиувилля для временно́й зависимости корреляционных функций в методе Мори [5]. С помощью функции $M_{\mathbf{q}}(\omega)$ решение уравнения (19) можно записать в виде

$$
\Phi_{\mathbf{q}}(\omega)=\frac{\chi_{\mathbf{q}}}{\omega-M_{\mathbf{q}}(\omega) / \chi_{\mathbf{q}}} .
$$

Для последовательного описания флуктуаций плотности заряда необходимо рассмотреть уравнение второго порядка для функции релаксации, составляя уравнение для функции памяти $M_{\mathbf{q}}\left(t-t^{\prime}\right)$, дважды дифференцируя ее по двум временам, $t$ и $t^{\prime}$. Вводя массовый оператор $\Sigma_{\mathbf{q}}(\omega)$ как неприводимую часть матрицы рассеяния в уравнении для $M_{\mathbf{q}}(\omega)$, как в уравнении $(19)$, решение для нее запишем в виде

$$
M_{\mathbf{q}}(\omega)=\frac{m_{\mathbf{q}}}{\omega-\Sigma_{\mathbf{q}}(\omega)} .
$$

Здесь неоднородный член в уравнении для $M_{\mathbf{q}}\left(t-t^{\prime}\right)$ имеет вид

$$
m_{\mathbf{q}}=\left(i \dot{N}_{\mathbf{q}} \mid-i \dot{N}_{-\mathbf{q}}\right)=\left\langle\left[i \dot{N}_{\mathbf{q}}, N_{-\mathbf{q}}\right]\right\rangle
$$

и массовый оператор $\Sigma_{\mathbf{q}}(\omega)$ определяется неприводимой частью функции релаксации "сил" $F_{\mathbf{q}}=(d / d t) j_{\mathbf{q}}=\ddot{N}_{\mathbf{q}}$ :

$$
\Sigma_{\mathbf{q}}(\omega)=\frac{1}{m_{\mathbf{q}}}\left(\left(F_{\mathbf{q}} \mid F_{-\mathbf{q}}\right)\right)_{\omega}^{(\mathrm{irr})} .
$$


Учитывая соотношения (22), (23), функцию релаксации запишем в виде

$$
\Phi_{\mathbf{q}}(\omega)=\chi_{\mathbf{q}} \frac{\omega-\Sigma_{\mathbf{q}}(\omega)}{\omega^{2}-\Omega_{\mathbf{q}}^{2}-\omega \Sigma_{\mathbf{q}}(\omega)},
$$

и согласно (17) для динамической зарядовой восприимчивости получим представление

$$
\chi_{\mathbf{q}}(\omega)=\chi_{\mathbf{q}}-\omega \Phi_{q}(\omega)=\frac{m_{\mathbf{q}}}{\Omega_{\mathbf{q}}^{2}+\omega \Sigma_{\mathbf{q}}(\omega)-\omega^{2}} .
$$

Здесь введена энергия зарядовых возбуждений в приближении среднего поля $\Omega_{\mathbf{q}}^{2}=$ $m_{\mathbf{q}} / \chi_{\mathbf{q}}$ и массовый оператор $\Sigma_{\mathbf{q}}(\omega)$ определяется функцией $(25)$.

2.3. Статическая восприимчивость. Получим выражение для статической зарядовой восприимчивости $\chi_{\mathbf{q}}=m_{\mathbf{q}} / \Omega_{\mathbf{q}}^{2}$ в выражении (27). Для ее вычисления воспользуемся соотношением

$$
\left(i \dot{N}_{\mathbf{q}} \mid-i \dot{N}_{-\mathbf{q}}\right)=\left(-\ddot{N}_{\mathbf{q}}, N_{-\mathbf{q}}\right)
$$

Корреляционная функция (24) в левой части уравнения находится прямым вычислением коммутатора (см. приложение А):

$$
\begin{aligned}
m_{\mathbf{q}} & =\left\langle\left[i \dot{N}_{\mathbf{q}}, N_{-\mathbf{q}}\right]\right\rangle=\sum_{\left(\mathbf{r}_{\mathbf{i}}-\mathbf{r}_{\mathbf{j}}\right)} e^{-i \mathbf{q}\left(\mathbf{r}_{\mathbf{i}}-\mathbf{r}_{\mathbf{j}}\right)}\left\langle\left[\left[N_{i}, H\right], N_{j}\right]\right\rangle= \\
& =-\frac{4}{N} \sum_{\mathbf{q}^{\prime}}\left[t\left(\mathbf{q}^{\prime}\right)-t\left(\mathbf{q}^{\prime}-\mathbf{q}\right)\right]\left\langle X_{\mathbf{q}^{\prime}}^{\sigma 0} X_{\mathbf{q}^{\prime}}^{0 \sigma}\right\rangle
\end{aligned}
$$

Для правой части соотношения (28) мы используем приближение среднего поля

$$
\left(-\ddot{N}_{\mathbf{q}}, N_{-\mathbf{q}}\right)=\left(\left[\left[N_{\mathbf{q}}, H\right], H\right], N_{-\mathbf{q}}\right) \approx \Omega_{\mathbf{q}}^{2}\left(N_{\mathbf{q}}, N_{-\mathbf{q}}\right),
$$

совершая расцепление высших корреляционных функций (см. приложение А). В результате получаем следующее представление для энергии зарядовых возбуждений:

$$
\begin{aligned}
\Omega_{\mathbf{q}}^{2}=\frac{\left(-\ddot{N}_{\mathbf{q}}, N_{-\mathbf{q}}\right)}{\left(N_{\mathbf{q}}, N_{-\mathbf{q}}\right)}=\sum_{\mathbf{q}^{\prime}} & \left.t\left(\mathbf{q}^{\prime}\right)-t\left(\mathbf{q}-\mathbf{q}^{\prime}\right)\right] \times \\
& \times\left(t\left(\mathbf{q}^{\prime}\right)+\frac{1}{2} J(\mathbf{q})-2 V(\mathbf{q})\right)\left\langle X_{\mathbf{q}^{\prime}}^{\sigma 0} X_{\mathbf{q}^{\prime}}^{0 \sigma}\right\rangle+\text { э.c. }
\end{aligned}
$$

Отметим, что в длинноволновом пределе $q \rightarrow 0$ спектр зарядовых возбуждений характеризуется акустической модой, $\Omega_{\mathbf{q}} \propto q$, если кулоновское взаимодействие в решетке $V(\mathbf{q})$ не имеет особенностей при $q \rightarrow 0$. При этом однородная статическая восприимчивость $\chi(0)=\chi_{\mathbf{q} \rightarrow \mathbf{0}}=\left(m_{\mathbf{q}} / \Omega_{\mathbf{q}}^{2}\right)_{\mathbf{q} \rightarrow \mathbf{0}}$ имеет конечное значение, поскольку в этом пределе $m_{\mathbf{q}} \propto q^{2}$ и $\Omega_{\mathbf{q}}^{2} \propto q^{2}$. Выражение для нее приводится в приложении А в пределе $q_{x}=q_{y} \rightarrow 0$ (формула (40)) и в пределе $q_{x} \rightarrow 0, q_{y}=0$ (формула (41)).

Рассмотрим поведение корреляционной функции флуктуаций заряда $\left\langle N_{\mathbf{q}} N_{-\mathbf{q}}\right\rangle$. В приближении среднего поля, в котором можно пренебречь вкладом массового 
оператора (25) в выражении для динамической зарядовой восприимчивости (27), корреляционная функция определяется уравнением

$$
\left\langle N_{\mathbf{q}} N_{-\mathbf{q}}\right\rangle=\int_{-\infty}^{\infty} \frac{d \omega}{e^{\omega / T}-1} \frac{1}{\pi} \operatorname{Im} \chi(\mathbf{q}, \omega+i \epsilon)=\frac{m_{\mathbf{q}}}{2 \Omega_{\mathbf{q}}} \operatorname{cth} \frac{\Omega_{\mathbf{q}}}{2 T} .
$$

В классическом пределе $\Omega_{\mathbf{q}} \ll T$ получаем стандартное соотношение между корреляционной функцией и статической восприимчивостью: $\left\langle N_{\mathbf{q}} N_{-\mathbf{q}}\right\rangle=T \chi_{\mathbf{q}}$. B квантовом пределе для $T=0$ находим $\left\langle N_{\mathbf{q}} N_{-\mathbf{q}}\right\rangle=m_{\mathbf{q}} / 2 \Omega_{\mathbf{q}}$, а в длинноволновом пределе $\left[m_{\mathbf{q}} / 2 \Omega_{\mathbf{q}}\right]_{q \rightarrow 0}=0$.

\section{3. РЕЗУЛЬТАТЫ}

Рассмотрим результаты, полученные для спектра зарядовых возбуждений (31) и статической восприимчивости $\chi_{\mathbf{q}}=m_{\mathbf{q}} / \Omega_{\mathbf{q}}^{2}$. Чтобы получить спектр электронных возбуждений в виде, соответствующем вычисленному в модели Хаббарда [37], [38], выберем параметр перескока в уравнении (2) в виде $t^{\prime}=0.1 t, t^{\prime \prime}=0.2 t$, где в качестве единицы энергии примем $t=0.4$ эВ. Как показывают модельные расчеты [39], межузельное кулоновское взаимодействие в купратах достаточно мало, так что параметры в (3) мы можем выбрать в виде $V_{1}=0.3 t$ и $V_{2}=0.2 t$. Однако в расчетах мы будем их варьировать при определении неустойчивости в модели к образованию ВЗП. Для обменного взаимодействия (4) примем стандартное значение $J=0.4 t$.

Для проведения расчетов необходимо также определить корреляционную функцию для дырок $\left\langle X_{\mathbf{q}}^{\sigma 0} X_{\mathbf{q}}^{0 \sigma}\right\rangle$ в формулах $(29),(31)$. Для нее используется следующее представление в приближении среднего поля:

$$
\left\langle X_{\mathbf{q}}^{\sigma 0} X_{\mathbf{q}}^{0 \sigma}\right\rangle=N_{\sigma}(\mathbf{q})=\frac{Q}{e^{\varepsilon(\mathbf{q}) / T}+1},
$$

где параметр $Q=1-n / 2$ описывает сужение одночастичной подзоны Хаббарда. Спектр дырочных возбуждений $\varepsilon(\mathbf{q})$ приводится в приложении Б (формулы (46), (47) и на рис. 8,9$)$. Для определения химического потенциала $\mu$ как функции концентрации дырок $n$ используется уравнение (7):

$$
n=\frac{1}{N} \sum_{\mathbf{q}, \sigma} N_{\sigma}(\mathbf{q})=\frac{1}{N} \sum_{\mathbf{q}} \frac{2-n}{e^{\varepsilon(\mathbf{q}) / T}+1} .
$$

Спектр зарядовых возбуждений $\Omega_{\mathbf{q}}^{2}$ вдоль основных направлений в зоне Бриллюэна $\Gamma(0,0) \rightarrow X(\pi, 0) \rightarrow M(\pi, \pi) \rightarrow \Gamma(0,0)$ показан на рис. 1 для разных значений концентрации дырок от $n=0.05$ до $n=0.3$ при стандартных параметрах модели $V_{1}=0.3$ и $V_{2}=0.2$ в формуле (3) и температуре $T=0.02 t \sim 90 \mathrm{~K}$. В центре зоны Бриллюэна $\Gamma(0,0)$ частота возбуждений обращается в нуль, $\Omega_{\mathbf{q}}^{2} \propto q^{2}$. На границе зоны Бриллюэна в точке $M(\pi, \pi)$ наблюдается максимум, интенсивность которого пропорциональна концентрации дырок. Дисперсия спектра зависит от параметров модели. Особенно сильное влияние на форму спектра оказывает изменение параметров кулоновского взаимодействия $V_{1}$ и $V_{2}$, что отражается на поведении статической восприимчивости, рассмотренной ниже. 


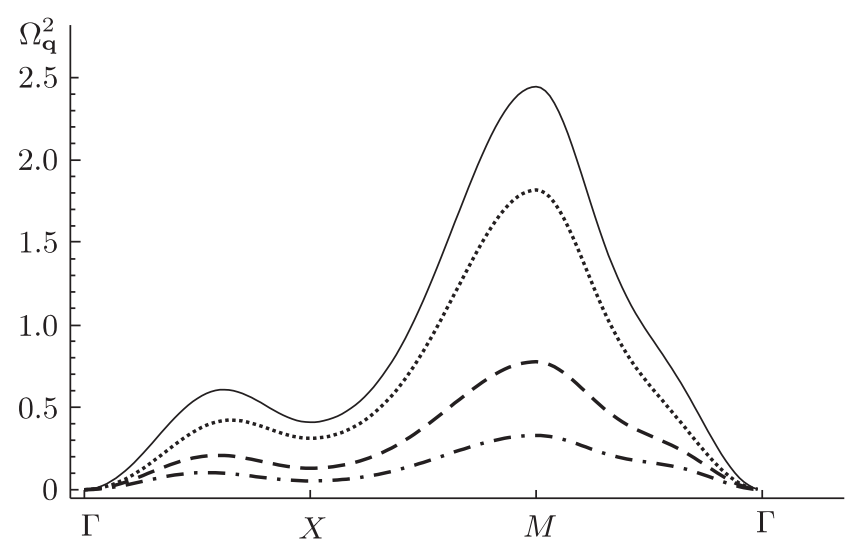

Рис. 1. Спектр зарядовых возбуждений $\Omega_{\mathbf{q}}^{2}$ вдоль основных направлений в зоне Бриллюэна: $\Gamma(0,0) \rightarrow X(\pi, 0) \rightarrow M(\pi, \pi) \rightarrow \Gamma(0,0)$ при концентрации дырок $n=0.05$ (штрихпунктирная линия), 0.1 (штриховая линия), 0.2 (пунктирная линия), 0.3 (сплошная линия).

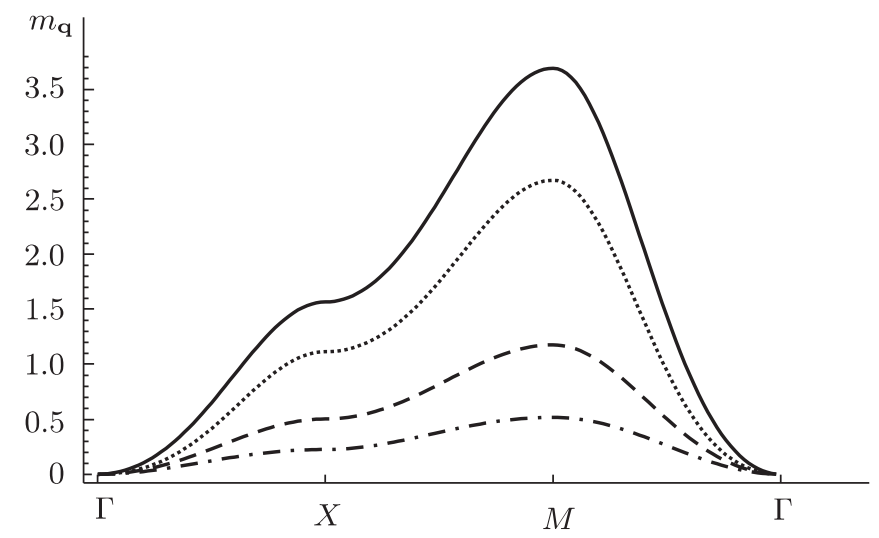

Рис. 2. Корреляционная функция $m_{\mathbf{q}}$ вдоль основных направлений в зоне Бриллюэна: $\Gamma(0,0) \rightarrow X(\pi, 0) \rightarrow M(\pi, \pi) \rightarrow \Gamma(0,0)$ при концентрации дырок $n=0.05$ (штрихпунктирная линия), 0.1 (штриховая линия), 0.2 (пунктирная линия), 0.3 (сплошная линия).

Зависимость концентрации корреляционной функции $m_{\mathbf{q}}$, представленной на рис. 2 , подобна зависимости спектра $\Omega_{\mathbf{q}}^{2}$ от $n$. В результате этого изменение статической восприимчивости $\chi_{\mathbf{q}}$ с изменением концентрации дырок оказывается несущественным (см. рис. 3 ). В то время как спектр $\Omega_{\mathbf{q}}^{2}$ и функция $m_{\mathbf{q}}$ имеют максимум в точке $M(\pi, \pi)$ зоны Бриллюэна, максимум $\chi_{\mathbf{q}}$ возникает в точке $X(\pi, 0)$. Заметим, что в работе [28] максимум статической восприимчивости наблюдался вблизи точки $M(\pi, \pi)$. Положение максимума, однако, может меняться, как обсуждается ниже. Вычисление однородной статической восприимчивости $\chi(0)=\chi_{\mathbf{q} \rightarrow \mathbf{0}}$ в пределе $q_{x}=q_{y} \rightarrow 0, \chi_{(11)}(0)(40)$, и в пределе $q_{x} \rightarrow 0, q_{y}=0, \chi_{(10)}(0)(41)$, дает одинаковые результаты: $\chi(0)$ слабо зависит от концентрации дырок, уменьшаясь 


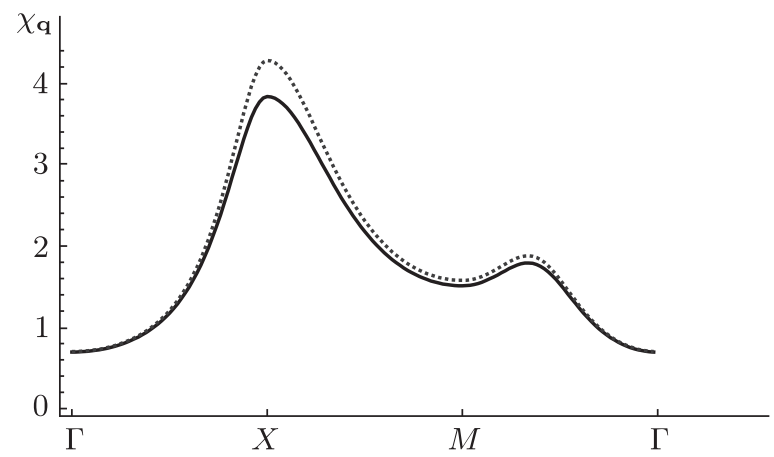

Рис. 3. Статическая восприимчивость $\chi_{\mathbf{q}}$ при концентрации дырок $n=0.05$ (пунктирная линия), $n=0.3$ (сплошная линия).

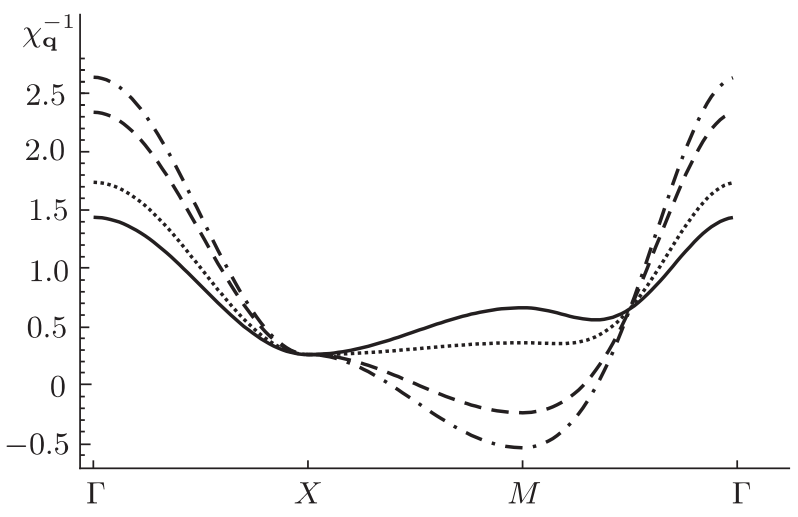

Рис. 4. Обратная статическая восприимчивость $\chi_{\mathbf{q}}^{-1}$ для $n=0.3$ при $V_{2}=0.2$ в зависимости от параметра $V_{1}: V_{1}=0.3$ (сплошная линия), $V_{1}=0.45$ (пунктирная линия), $V_{1}=0.75$ (штриховая линия), $V_{1}=0.9$ (штрихпунктирная линия).

с ростом концентрации дырок от значения $\chi(0)=0.70$ при $n=0.05$ до $\chi(0)=0.68$ при $n=0.2$ и увеличиваясь далее до $\chi(0)=0.69$ при $n=0.3$.

При определенных параметрах модели статическая восприимчивость неограниченно возрастает, что указывает на неустойчивость однородного распределения заряда и образование ВЗП. Для анализа этой неустойчивости удобно рассмотреть обратную восприимчивость $\chi_{\mathbf{q}}^{-1}$, обращение которой в нуль означает переход в неоднородное состояние. На рис. 4 показана зависимость $\chi_{\mathbf{q}}^{-1}$ для разных значений параметра $V_{1}$ при фиксированной величине $V_{2}=0.2$ для концентрации дырок $n=0.3$. При увеличении параметра $V_{1}$ пик в статической восприимчивости (минимум в $\chi_{\mathbf{q}}^{-1}$ ) смещается из точки $X(\pi, 0)$ в точку $M(\pi, \pi)$. При значении $V_{1}=0.75$ обратная восприимчивость становится отрицательной вблизи этой точки, что свидетельствует о неустойчивости однородного распределения заряда и о возникновении ВЗП с волновым вектором $Q_{x}=Q_{y} \approx 0.3(\pi / a)$. С ростом параметра $V_{1}$ волновой вектор увеличивается. Столь же сильная вариация наблюдается для $\chi_{\mathbf{q}}^{-1}$ при изменении 


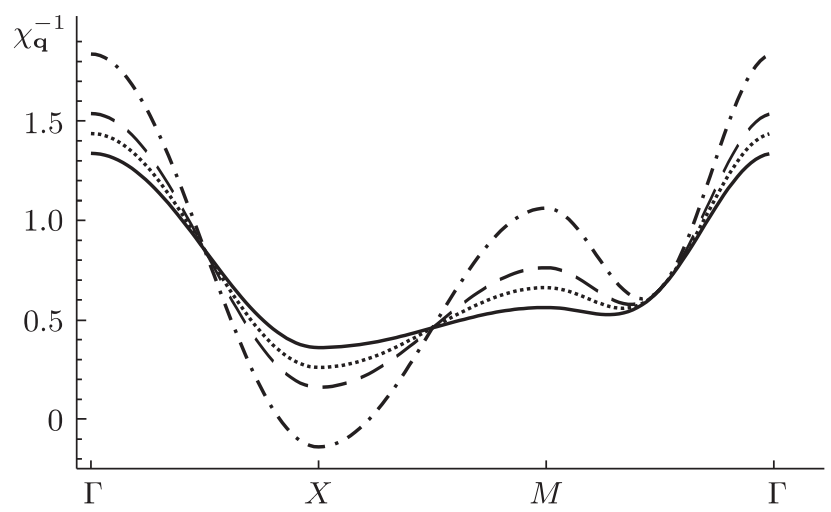

Рис. 5. Обратная статическая восприимчивость $\chi_{\mathbf{q}}^{-1}$ для $n=0.3$ при $V_{1}=0.3$ в зависимости от параметра $V_{2}: V_{2}=0.15$ (сплошная линия), $V_{2}=0.2$ (пунктирная линия), $V_{2}=0.25$ (штриховая линия), $V_{2}=0.4$ (штрихпунктирная линия).

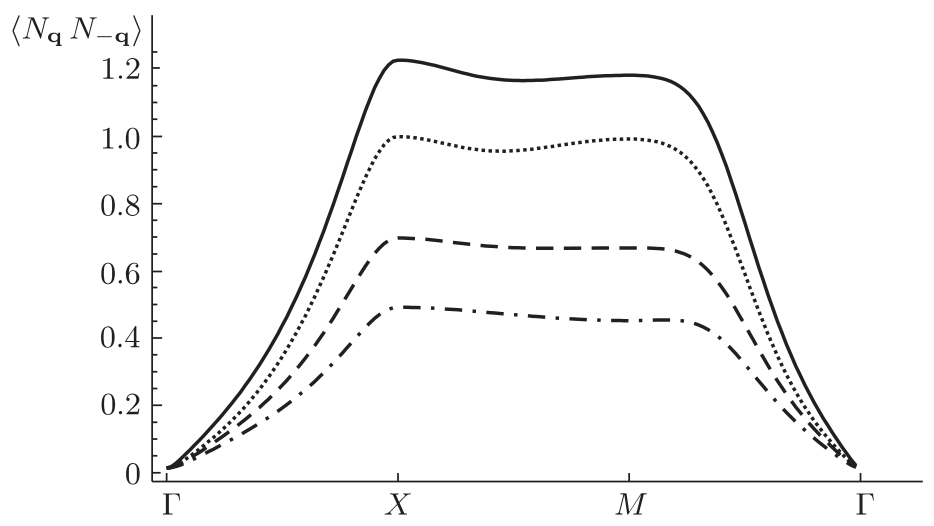

Рис. 6. Корреляционная функция $\left\langle N_{\mathbf{q}} N_{-\mathbf{q}}\right\rangle$ при концентрации дырок $n=0.05$ (штрихпунктирная линия), 0.1 (штриховая линия), 0.2 (пунктирная линия), 0.3 (сплошная линия).

параметра $V_{2}$ при фиксированном значении $V_{1}=0.3$ при $n=0.3$, как это демонстрируется на рис. 5. При увеличении параметра $V_{2}$ до значения $V_{2}=0.4$ возникает ВЗП вблизи точки $X(\pi, 0)$ с волновым вектором $Q_{x} \approx 0.12(\pi / a)$. Таким образом, в зависимости от параметров кулоновского взаимодействия $V_{1}$ и $V_{2}$, которые имеют различную симметрию согласно (3), ВЗП может возникать либо вдоль направления $\Gamma(0,0) \rightarrow M(\pi, \pi)$ при увеличении параметра $V_{1}$, либо вдоль направления $\Gamma(0,0) \rightarrow X(\pi, 0)$ при увеличении параметра $V_{2}$.

Изменение корреляционной функции флуктуаций заряда $\left\langle N_{\mathbf{q}} N_{-\mathbf{q}}\right\rangle$ (32) с концентрацией дырок представлена на рис. 6 для стандартных параметров модели. По сравнению со статической восприимчивостью $\chi_{\mathbf{q}}=m_{\mathbf{q}} / \Omega_{\mathbf{q}}^{2}$, эта функция растет быстрее с концентрацией дырок за счет более быстрого роста $m_{\mathbf{q}}$ по сравнению с $\Omega_{\mathbf{q}}$ в формуле (32). Зависимость функции от волнового вектора подобна 


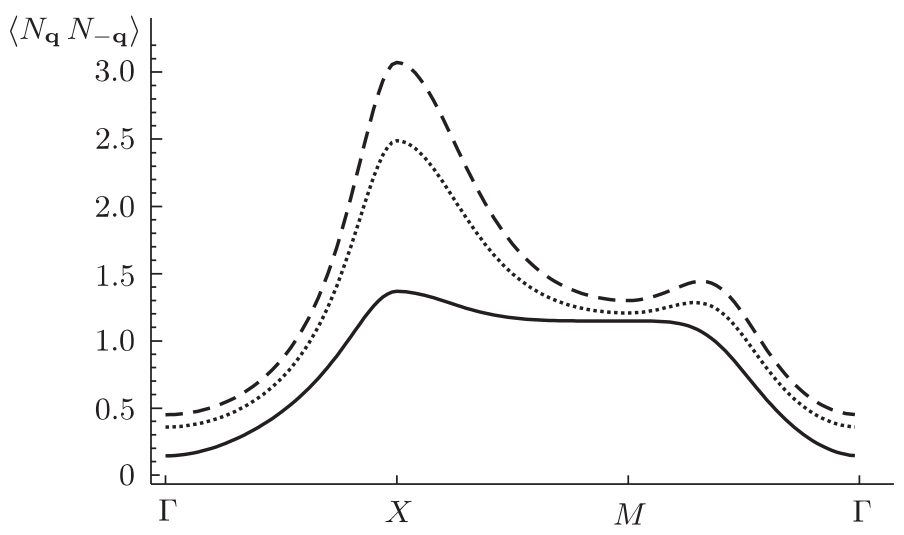

Рис. 7. Корреляционная функция $\left\langle N_{\mathbf{q}} N_{-\mathbf{q}}\right\rangle$ при концентрации дырок $n=0.3$ в зависимости от температуры: $T=0.02$ (сплошная линия), 0.48 (пунктирная линия), 0.6 (штриховая линия).

дисперсии $\chi_{\mathbf{q}}$, особенно при высоких температурах в классическом пределе, когда $\left\langle N_{\mathbf{q}} N_{-\mathbf{q}}\right\rangle=T \chi_{\mathbf{q}}$, как это показано на рис. 7. При этом температурная зависимость статической восприимчивости оказывается слабой ввиду слабой зависимости функций $m_{\mathbf{q}}$ и $\Omega_{\mathbf{q}}^{2}$ от температуры, которая определяется распределением Ферми в корреляционной функции $N_{\sigma}(\mathbf{q})(33)$.

\section{4. ЗАКЛЮЧЕНИЕ}

В настоящей работе мы рассмотрели поведение статической восприимчивости $\chi_{\mathbf{q}}$ в системе электронов с сильной корреляцией в рамках $t-J-V$ модели при небольшой концентрации дырок. В отличие от других работ (см., например, [22]-[24], [28]), где динамическая восприимчивость вычисляется в приближении типа случайных фаз, когда оператор плотности заряда представляется в виде произведения одночастичных операторов (в операторах Хаббарда: $X_{i}^{\sigma \sigma}=X_{i}^{\sigma 0} X_{i}^{0 \sigma}$ ), в нашей работе мы использовали исходное представление для оператора плотности заряда $N_{\mathbf{q}}$ и вычислили коллективную ФГ от этих операторов, пользуясь методом функции релаксации. Было показано, что при достаточно сильном межузельном кулоновском отталкивании дырок величина $\chi_{\mathbf{q}}$ неограниченно возрастает $\left(\chi_{\mathbf{q}}^{-1}\right.$ обращается в нуль) и в системе появляется ВЗП либо вдоль диагонали элементарной ячейки (вблизи точки $M(\pi, \pi)$ зоны Бриллюэна), либо вдоль ребра элементарной ячейки (вблизи точки $X(\pi, 0)$ зоны Бриллюэна). Зависимость от других параметров модели, в том числе от обменного антиферромагнитного взаимодействия $J$, более слабая. Статическая восприимчивость слабо зависит от концентрации дырок и существенно не меняется с температурой, в отличие от корреляционной функции флуктуаций заряда $\left\langle N_{\mathbf{q}} N_{-\mathbf{q}}\right\rangle$. В рамках $t-J-V$ модели, не рассматривающей внутреннюю структуру элементарной ячейки $\mathrm{CuO}_{2}$, невозможно описать появление ВЗП на узлах кислорода, наблюдаемой в экспериментах. Для уточнения полученных результатов и анализа частотной зависимости динамической восприимчивости необходимо рассмотреть массовый оператор в общем представлении для $\chi_{\mathbf{q}}(\omega)(27)$, что предполагается сделать в следующей работе. 


\section{Вычисление $\Omega_{\mathbf{q}}^{2}$}

\section{ПРИЛОЖЕНИЕ А}

Корреляционная функция $m_{\mathbf{q}}$ определяется фурье-компонентой коммутатора

$$
\begin{aligned}
m_{i j} & =\left\langle\left[\left[N_{i}, H\right], N_{j}\right]\right\rangle=\sum_{k, \sigma} t_{i k}\left\langle\left[\left(X_{i}^{\sigma 0} X_{k}^{0 \sigma}-X_{k}^{\sigma 0} X_{i}^{0 \sigma}\right), N_{j}\right]\right\rangle= \\
& =\sum_{\sigma} t_{i j}\left\langle X_{i}^{\sigma 0} X_{j}^{0 \sigma}+X_{j}^{\sigma 0} X_{i}^{0 \sigma}\right\rangle-\delta_{i, j} \sum_{k, \sigma} t_{i k}\left\langle X_{i}^{\sigma 0} X_{k}^{0 \sigma}+X_{k}^{\sigma 0} X_{i}^{0 \sigma}\right\rangle,
\end{aligned}
$$

где обменное и кулоновское взаимодействия вкладов не дают. Вводя фурье-представления для параметра перескока $t(\mathbf{q})(40)$ и корреляционной функции $\left\langle X_{\mathbf{q}}^{\sigma 0} X_{\mathbf{q}}^{0 \sigma}\right\rangle$ (33), получаем формулу для $m_{\mathbf{q}}(29)$.

Для вычисления спектра зарядовых возбуждений $\Omega_{\mathbf{q}}^{2}$ согласно соотношению (30) необходимо вычислить вторую производную от оператора $-\ddot{N}_{\mathbf{q}}=\left[\left[N_{\mathbf{q}}, H\right], H\right]$. Выполняя последовательные коммутации для оператора числа частиц $N_{i}$ с гамильтонианом $H(1)$, получаем

$$
\begin{aligned}
-\ddot{N}_{i}= & \sum_{m j, \sigma \sigma^{\prime}} t_{i m}\left[\left(t_{m j} X_{i}^{\sigma 0} B_{m}^{\sigma^{\prime} \sigma} X_{j}^{0 \sigma^{\prime}}-t_{i j} X_{m}^{\sigma 0} B_{i}^{\sigma^{\prime} \sigma} X_{j}^{0 \sigma^{\prime}}\right)+\text { э.c. }\right]- \\
& -\frac{1}{2} \sum_{m j, \sigma \sigma^{\prime}} t_{i m}\left[\left(J_{m j} X_{i}^{\sigma 0} X_{m}^{0 \sigma^{\prime}}-J_{i j} X_{m}^{\sigma 0} X_{i}^{0 \sigma^{\prime}}\right)\left(\delta_{\sigma^{\prime}, \sigma}-1\right) B_{j}^{\sigma^{\prime} \sigma}+\text { э.c. }\right]- \\
& -\sum_{m j, \sigma} t_{i m}\left[\left(V_{i j}-V_{j m}\right) N_{j} X_{i}^{\sigma 0} X_{m}^{0 \sigma}+\text { э.c. }\right],
\end{aligned}
$$

где

$$
B_{i}^{\sigma^{\prime} \sigma}=\left(X_{i}^{00}+X_{i}^{\sigma \sigma}\right) \delta_{\sigma^{\prime} \sigma}+X_{i}^{\bar{\sigma} \sigma} \delta_{\sigma^{\prime} \bar{\sigma}}=\left[1-\frac{1}{2} N_{i}+\sigma S_{i}^{z}\right] \delta_{\sigma \sigma^{\prime}}+S_{i}^{\bar{\sigma}} \delta_{\bar{\sigma} \sigma^{\prime}}
$$

Составляя скалярное произведение $\left(-\ddot{N}_{i}, N_{k}\right)$ функции (36), мы произведем расцепление корреляционных функций, учитывая в скалярном произведении только члены, содержащие оператор числа частиц, например

$$
\sum_{m j, \sigma \sigma^{\prime}} t_{i m} t_{m j}\left(X_{i}^{\sigma 0} B_{m}^{\sigma^{\prime} \sigma} X_{j}^{0 \sigma^{\prime}} N_{k}\right)=-\frac{1}{2} \sum_{m j, \sigma} t_{i m} t_{m j}\left\langle X_{i}^{\sigma 0} X_{j}^{0 \sigma}\right\rangle\left(N_{m}, N_{k}\right) .
$$

Вводя фурье-компоненты параметров перескока $t_{i j}$, взаимодействий $J_{i j}, V_{i j}$ и корреляционных функций, получим уравнение

$$
\begin{aligned}
\left(-\ddot{N}_{\mathbf{q}}, N_{-\mathbf{q}}\right)= & \sum_{\mathbf{q}^{\prime}}\left[t\left(\mathbf{q}^{\prime}\right)-t\left(\mathbf{q}-\mathbf{q}^{\prime}\right)\right] \times \\
& \times\left(t\left(\mathbf{q}^{\prime}\right)+\frac{1}{2} J(\mathbf{q})-2 V(\mathbf{q})\right)\left\langle X_{\mathbf{q}^{\prime}}^{\sigma 0} X_{\mathbf{q}^{\prime}}^{0 \sigma}\right\rangle\left(N_{\mathbf{q}}, N_{-\mathbf{q}}\right)+\text { э.c. }
\end{aligned}
$$

Учитывая уравнение (30), для спектра зарядовых возбуждений получим формулу (31).

Вычислим однородную статическую восприимчивость $\chi(0)=\chi_{\mathbf{q} \rightarrow \mathbf{0}}=\left(m_{\mathbf{q}} / \Omega_{\mathbf{q}}^{2}\right)_{\mathbf{q} \rightarrow \mathbf{0}}$ в двух пределах: при $q_{x}=q_{y} \rightarrow 0$, т. е. в направлении $M(\pi, \pi) \rightarrow \Gamma(0,0)$, и в другом 
направлении, $X(\pi, \pi) \rightarrow \Gamma(0,0)$, при $q_{x} \rightarrow 0, q_{y}=0$. В первом случае получаем

$$
\begin{aligned}
\chi_{(11)}(0)=\lim _{q_{x} \rightarrow 0} \frac{m_{\mathbf{q}}}{\Omega_{\mathbf{q}}^{2}}=-\frac{4}{N} \sum_{\mathbf{q}^{\prime}}\left[\left(t+2 t^{\prime}\right) \gamma\left(\mathbf{q}^{\prime}\right)+4 t^{\prime \prime} \gamma^{\prime \prime}\left(\mathbf{q}^{\prime}\right)\right]\left\langle X_{\mathbf{q}^{\prime}}^{\sigma 0} X_{\mathbf{q}^{\prime}}^{0 \sigma}\right\rangle \times \\
\times\left\{\frac{1}{N} \sum_{\mathbf{q}^{\prime}}\left[\left(t+2 t^{\prime}\right) \gamma\left(\mathbf{q}^{\prime}\right)+4 t^{\prime \prime} \gamma^{\prime \prime}\left(\mathbf{q}^{\prime}\right)\right] \times\right. \\
\left.\quad \times\left(t\left(\mathbf{q}^{\prime}\right)+2 J-8\left(V_{1}+V_{2}\right)\right)\left\langle X_{\mathbf{q}^{\prime}}^{\sigma 0} X_{\mathbf{q}^{\prime}}^{0 \sigma}\right\rangle+\text { э.c. }\right\}^{-1} .
\end{aligned}
$$

Во втором случае находим

$$
\begin{aligned}
\chi_{(10)}(0)=\lim _{q_{x} \rightarrow 0} \frac{m_{\mathbf{q}}}{\Omega_{\mathbf{q}}^{2}}=-\frac{1}{N} \sum_{\mathbf{q}^{\prime}}\left[2 t \cos q_{x}^{\prime}+4 t^{\prime} \cos q_{y}^{\prime}+8 t^{\prime \prime} \cos 2 q_{x}^{\prime}\right]\left\langle X_{\mathbf{q}^{\prime}}^{\sigma 0} X_{\mathbf{q}^{\prime}}^{0 \sigma}\right\rangle \times \\
\times\left\{\frac{1}{2 N} \sum_{\mathbf{q}^{\prime}}\left[t \cos q_{x}^{\prime}+8 t^{\prime} \cos q_{y}^{\prime}+4 t^{\prime \prime} \cos 2 q_{x}^{\prime}\right] \times\right. \\
\left.\quad \times\left(t\left(\mathbf{q}^{\prime}\right)+2 J-8\left(V_{1}+V_{2}\right)\right)\left\langle X_{\mathbf{q}^{\prime}}^{\sigma 0} X_{\mathbf{q}^{\prime}}^{0 \sigma}\right\rangle+\text { э.c. }\right\}^{-1} .
\end{aligned}
$$

\section{Вычисление спектра $\varepsilon(\mathbf{q})$}

ПРИЛОЖЕНИЕ Б

Для вычисления электронного спектра в $t-J-V$ модели (1) мы воспользуемся методом проектирования для ФГ, следуя работе [34]. Рассмотрим одночастичную антикоммутаторную ФГ в представлении операторов Хаббарда

$$
G_{i j, \sigma}\left(t-t^{\prime}\right)=\left\langle\left\langle X_{i}^{0 \sigma}(t) \mid X_{j}^{\sigma 0}\left(t^{\prime}\right)\right\rangle\right\rangle
$$

где использованы обозначения Зубарева [3]. Фурье-представление в $(\mathbf{q}, \omega)$-пространстве определяется уравнением

$$
G_{i j, \sigma}\left(t-t^{\prime}\right)=\frac{1}{2 \pi} \int_{-\infty}^{\infty} d t e^{-i\left(t-t^{\prime}\right)} \frac{1}{N} \sum_{\mathbf{k}} e^{i \mathbf{q}(\mathbf{i}-\mathbf{j})} G_{\sigma}(\mathbf{q}, \omega) .
$$

В настоящей работе мы вычисляем электронный спектр в приближении среднего поля, пренебрегая вкладом массового оператора, вычисленным в работе [34]. В этом случае фурье-компонента $Ф Г ~(42)$ имеет вид

$$
G(\mathbf{q}, \omega)=Q \frac{1}{\omega-\varepsilon(\mathbf{q})} .
$$

Спектр дырочных возбуждений $\varepsilon(\mathbf{q})$ в приближении среднего поля определяется равновесной корреляционной функцией $\left\langle\left\{\left[X_{i}^{0 \sigma}, H\right], X_{j}^{\sigma 0}\right\}\right\rangle$ :

$$
\varepsilon(\mathbf{q})=\frac{1}{N} \sum_{\mathbf{j}} e^{i \mathbf{q}(\mathbf{i}-\mathbf{j})}\left\langle\left\{\left[X_{i}^{0 \sigma}, H\right], X_{j}^{\sigma 0}\right\}\right\rangle Q^{-1} .
$$

Выполняя коммутации и переходя к фурье-представлению, получим

$$
\begin{gathered}
\varepsilon(\mathbf{q})=4 t \alpha \gamma(\mathbf{q})+4 t^{\prime} \beta \gamma^{\prime}(\mathbf{q})+4 t^{\prime \prime} \beta \gamma^{\prime \prime}(\mathbf{q})-\omega^{(c)}(\mathbf{q})-\mu, \\
\omega^{(c)}(\mathbf{q})=\frac{1}{N} \sum_{\mathbf{k}, \sigma} V(\mathbf{q}-\mathbf{k}) N_{\sigma}(\mathbf{k}),
\end{gathered}
$$




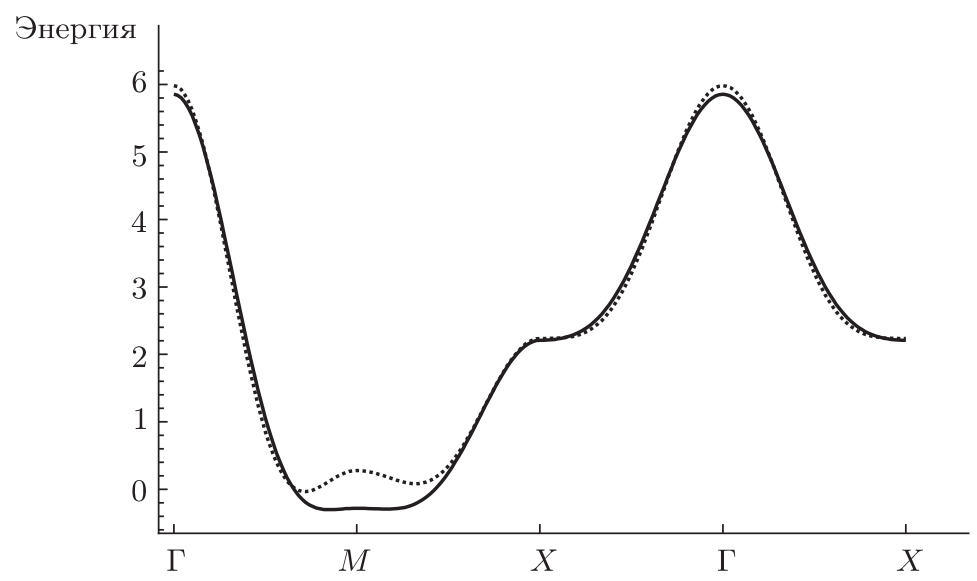

Рис. 8. Дисперсия электронного спектра вдоль основных направлений в зоне Бриллюэна: $\Gamma(0,0) \rightarrow X(\pi, 0) \rightarrow M(\pi, \pi) \rightarrow \Gamma(0,0)$ при концентрации дырок $n=0.05$ (пунктирная линия), 0.3 (сплошная линия).

где вклад $\omega^{(c)}(\mathbf{q})$ обусловлен кулоновским взаимодействием дырок и величина его мала для стандартных значений параметров $V_{1}=0.3$ и $V_{2}=0.2$. Перенормировка спектра (46) определяется функциями

$$
\alpha=Q\left[1+\frac{C_{1}}{Q^{2}}\right], \quad \beta=Q\left[1+\frac{C_{2}}{Q^{2}}\right],
$$

где перенормировка спектра за счет спиновых корреляций определяется корреляционными функциями первых и вторых соседей:

$$
C_{1}=\left\langle\mathbf{S}_{i} \mathbf{S}_{i \pm a_{x} / a_{y}}\right\rangle, \quad C_{2}=\left\langle\mathbf{S}_{i} \mathbf{S}_{i \pm a_{x} \pm a_{y}}\right\rangle .
$$

Для их вычисления мы использовали модельный подход, как и в работе [34], где зависимость функций $C_{1}$ и $C_{2}$ от концентрации дырок $n$ связана с изменением корреляционной длины $\xi$ ближнего антиферромагнитного порядка. В табл. 1 приведена эта зависимость и значения параметров перенормировки (48) согласно [34].

ТАБлицА 1. Антиферромагнитная корреляционная длина $\xi$, статические корреляционные функции $C_{1}, C_{2}$ и параметры перенормировки (48) при различных значениях концентрации дырок $n$.

\begin{tabular}{crrrr}
\hline$n$ & 0.05 & 0.10 & 0.20 & 0.30 \\
\hline$\xi$ & 3.40 & 2.50 & 1.70 & 1.40 \\
$C_{1}$ & -0.26 & -0.21 & -0.14 & -0.10 \\
$C_{2}$ & 0.16 & 0.11 & 0.06 & 0.04 \\
$\alpha$ & 0.71 & 0.73 & 0.74 & 0.73 \\
$\beta$ & 1.14 & 1.07 & 0.97 & 0.90 \\
\hline
\end{tabular}

Зависимость спиновых корреляционных функций от концентрации дырок определяет изменение электронного спектра с легированием, как это показано на рис. 8 . 


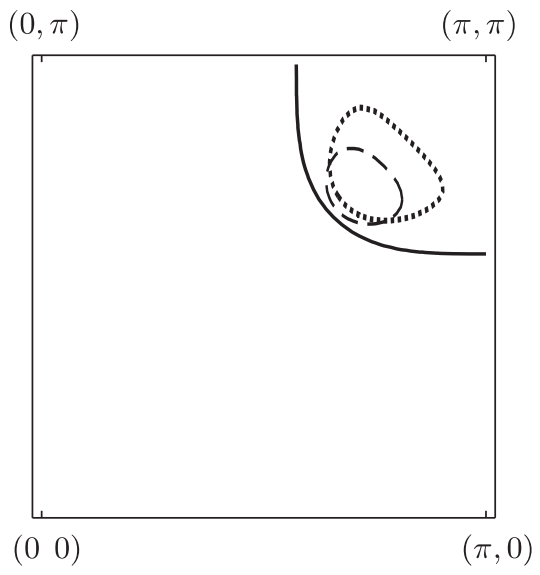

Рис. 9. Поверхность Ферми в четверти зоны Бриллюэна при концентрации дырок $n=0.05$ (штриховая линия), 0.1 (пунктирная линия), 0.3 (сплошная линия).

С увеличением концентрации дырок антиферромагнитные корреляции ослабевают и дисперсия приобретает вид, характерный для свободных электронов. При этом меняется и вид поверхности Ферми, которая определяется уравнением $\varepsilon\left(\mathbf{q}_{\mathrm{F}}\right)=$ $t\left(\mathbf{q}_{\mathrm{F}}\right)-\mu=0$ и представлена на рис. 9. При малой концентрации дырок она имеет вид четырех карманов вблизи точек $( \pm \pi / 2, \pm \pi / 2)$, а при увеличении концентрации она превращается в большую открытую поверхность Ферми. Близкие результаты были получены в модели Хаббарда для электронов [38], где хорошо прослеживается переход от малой поверхности Ферми к большой при увеличении концентрации дырок.

\section{Список литературы}

[1] Н.Н. Боголюбов, С. В. Тябликов, "Запаздывающие и опережающие функции Грина в статистической физике", Докл. АН СССР, 126 (1959), 53-58.

[2] С. В. Тябликов, Методы квантовой теории магнетизма, Наука, М., 1975.

[3] Д. Н. Зубарев, “Двухвременные функции Грина в статистической физике”, УФН, 71:1 (1960), 71-116.

[4] Д. Н. Зубарев, В. Г. Морозов, Г. Рёпке, Статистическая механика неравновесных процессов, т. 1, Физматлит, М., 2002.

[5] H. Mori, "A continued-fraction representation of the time-correlation functions", Prog. Theor. Phys., 34:3 (1965), 399-416.

[6] N. M. Plakida, "Projection operator method", Strongly Correlated Systems. Theoretical Methods, Springer Series in Solid-State Sciences, 171, eds. A. Avella, F. Mancini, Springer, Berlin, Heidelberg, 2011, 173-202.

[7] Н. М. Плакида, “Двухвременные функции Грина и диаграммная техника”, ТМФ, 168:3 (2011), 518-535.

[8] A. A. Vladimirov, D. Ihle, N. M. Plakida, "Dynamic spin susceptibility in the $t-J$ model", Phys. Rev. B, 80:10 (2009), 104425, 12 pp.

[9] A. A. Vladimirov, D. Ihle, N. M. Plakida, "Dynamic spin susceptibility of superconducting cuprates: a microscopic theory of the magnetic resonance mode", Phys. Rev. B, 83:1 (2011), 024411, 13 pp. 
[10] A. A. Vladimirov, D. Ihle, N. M. Plakida, "Optical and dc conductivities of cuprates: spin-fluctuation scattering in the $t-J$ model", Phys. Rev. B, 85:22 (2012), 224536, 12 pp.

[11] S. Blanco-Canosa, A. Frano, E. Schierle, J. Porras, T. Loew, M. Minola, M. Bluschke, E. Weschke, B. Keimer, M. Le Tacon1, "Resonant x-ray scattering study of charge-density wave correlations in $\mathrm{YBa}_{2} \mathrm{Cu}_{3} \mathrm{O}_{6+x}$ ", Phys. Rev. B, 90:5 (2014), 054513, 13 pp.

[12] M. Hücker, N. B. Christensen, A. T. Holmes, E. Blackburn, E. M. Forgan, Ruixing Liang, D. A. Bonn, W. N. Hardy, O. Gutowski, M. v. Zimmermann, S. M. Hayden, J. Chang, "Competing charge, spin, and superconducting orders in underdoped $\mathrm{YBa}_{2} \mathrm{Cu}_{3} \mathrm{O}_{y}$ ", Phys. Rev. B, 90:5 (2014), 054514, 11 pp.

[13] R. Comin, R. Sutarto, F. He, E. H. da Silva Neto, L. Chauviere, A. Frano, R. Liang, W. N. Hardy, D. A. Bonn, Y. Yoshida, H. Eisaki, A. J. Achkar, D. G. Hawthorn, B. Keimer, G. A. Sawatzky, A. Damascelli, "Symmetry of charge order in cuprates", Nature Materials, 14 (2015), 796-800.

[14] R. Comin, R. Sutarto, E. H. da Silva Neto, L. Chauviere, R. Liang, W. N. Hardy, D. A. Bonn, F. He, G. A. Sawatzky, A. Damascelli, "Broken translational and rotational symmetry via charge stripe order in underdoped $\mathrm{YBa}_{2} \mathrm{Cu}_{3} \mathrm{O}_{6+y}$ ", Science, 347:6228 (2015), 1335-1339.

[15] R. Comin, A. Frano, M. M. Yee, Y. Yoshida, H. Eisaki, E. Schierle, E. Weschke, R. Sutarto, F. He, A. Soumyanarayanan, Y. He, M. Le Tacon, I. S. Elfimov, J. E. Hoffman, G. A. Sawatzky, B. Keimer, A. Damascelli, "Charge order driven by Fermi-arc instability in $\mathrm{Bi}_{2} \mathrm{Sr}_{2-x} \mathrm{La}_{x} \mathrm{CuO}_{6+d}$ ", Science, 343:6169 (2014), 390-392.

[16] M. Hashimoto, G. Ghiringhelli, W.-S. Lee, G. Dellea, A. Amorese, C. Mazzoli, K. Kummer, N.B. Brookes, B. Moritz, Y. Yoshida, H. Eisaki, Z. Hussain, T. P. Devereaux, Z.-X. Shen, L. Braicovich, "Direct observation of bulk charge modulations in optimally doped $\mathrm{Bi}_{1.5} \mathrm{~Pb}_{0.6} \mathrm{Sr}_{1.54} \mathrm{CaCu}_{2} \mathrm{O}_{8+\delta}$ ", Phys. Rev. B, 89:22 (2014), 220511, 5 pp.

[17] E. H. da Silva Neto, P. Aynajian, A. Frano, R. Comin, E. Schierle, E. Weschke, A. Gyenis, J. Wen, J. Schneeloch, Z. Xu, S. Ono, G. Gu, M. Le Tacon, A. Yazdani, "Ubiquitous interplay between charge ordering and high-temperature superconductivity in cuprates", Science, 343:6169 (2014), 393-396.

[18] M. H. Hamidian, S. D. Edkins, C. K. Kim, J. C. Davis, A. P. Mackenzie, H. Eisaki, S. Uchida, M. J. Lawler, E.-A. Kim, S. Sachdev, K. Fujita, "Atomic-scale electronic structure of the cuprate $d$-symmetry form factor density wave state", Nat. Phys., 12 (2016), 150-155.

[19] W. Tabis, Y. Li, M. Le Tacon, L. Braicovich, A. Kreyssig, M. Minola, G. Dellea, E. Weschke, M. J. Veit, M. Ramazanoglu, A.I. Goldman, T. Schmitt, G. Ghiringhelli, N. Barisic, M. K. Chan, C. J. Dorow, G. Yu, X. Zhao, B. Keimer, M. Greven, "Charge order and its connection with Fermi-liquid charge transport in a pristine high- $T_{\mathrm{c}}$ cuprate", Nature Commun., 5 (2014), 5875, 6 pp.

[20] G. Campi, A. Bianconi, N. Poccia, G. Bianconi, L. Barba, G. Arrighetti, D. Innocenti, J. Karpinski, N. D. Zhigadlo, S. M. Kazakov, M. Burghammer, M. v. Zimmermann, M. Sprung, A. Ricci, "Inhomogeneity of charge-density-wave order and quenched disorder in a high- $T$ + c superconductor", Nature, 525 (2015), 359-362.

[21] P. A. Volkov, K. B. Efetov, "Spin-fermion model with overlapping hot spots and charge modulation in cuprates", Phys. Rev. B, 93:8 (2016), 085131, 21 pp.

[22] I. Eremin, M. Eremin, S. Varlamov, D. Brinkmann, M. Mali, J. Roos, "Spin susceptibility and pseudogap in $\mathrm{YBa}_{2} \mathrm{Cu}_{4} \mathrm{O}_{8}$ : an approach via a charge-density-wave instability", Phys. Rev. B, 56:17 (1997), 11305-11311.

[23] С. В. Варламов, М. В. Еремин, И. М. Еремин, "К теории псевдощели в спектре элементарных возбуждений нормальной фазе бислойных купратов", Писъма в ЖЖЭТФ, 66:8 (1997), 533-538.

[24] M. Eremin, S. Varlamov, I. Eremin, "Magnitude of spin and charge density wave amplitudes in underdoped cuprates", Appl. Magn. Reson., 19:3-4 (2000), 355-362.

[25] М.В. Еремин, М.А. Малахов, “Эффективное кулоновское взаимодействие электронов в купратах", Изв. РАН. Сер. физ., 78:9 (2014), 1183-1186. 
[26] М. В. Еремин, М. А. Малахов, “Динамическая зарядовая восприимчивость и смягчение продольной фононной моды в купратах”, Писъма в ЖЭТФ, 100:5 (2014), 362-365.

[27] М. В. Еремин, Д.А. Сюняев, “Температурная зависимость глубины проникновения магнитного поля при наличии дисперсии у параметров порядка сверхпроводимости и волн зарядовых плотностей”, Писъма в ЖЖЭТФ, 103:3 (2016), 209-213.

[28] M. Eremin, I. Eremin, S. Varlamov, "Dynamical charge susceptibility in layered cuprates: beyond the conventional random-phase-approximation scheme", Phys. Rev. B, 64:21 (2001), 214512, 7 pp.

[29] E. Bulut, " $d_{x^{2}-y^{2}}$ superconductivity and the Hubbard model", Adv. Phys., 51:7 (2002), $1587-1667$.

[30] W. Götze, P. Wölfle, "Homogeneous dynamical conductivity of simple metals", Phys. Rev. B, 6:4 (1972), 1226-1238.

[31] D. Forster, Hydrodynamic Fluctuations, Broken Symmetry and Correlation Functions, Benjamin, New York, 1975.

[32] Ю.А. Церковников, "О методе решения бесконечных систем уравнений для двухвременных температурных функций Грина”, ТМФ, 49:2 (1981), 219-233.

[33] G. Jackeli, N. M. Plakida, "Charge dynamics and optical conductivity of the $t-J$ model", Phys. Rev. B, 60:8 (1999), 5266-5275.

[34] N.M. Plakida, V.S. Oudovenko, "Electron spectrum and superconductivity in the $t-J$ model at moderate doping", Phys. Rev. B, 59:18 (1999), 11949-11961.

[35] J. Hubbard, "Electron correlations in narrow energy bands. IV. The atomic representation", Proc. Roy. Soc. Ser. A, 285 (1965), 542-560.

[36] Ю.А. Изюмов, Ю.Н. Скрябин, Статистическая механика магнитоупорядоченных систем, Наука, М., 1987.

[37] N.M. Plakida, V.S. Oudovenko, "Electronic spectrum in high-temperature cuprate superconductors", ЖЭТФ, 131:2 (2007), 259-274.

[38] N. M. Plakida, V. S. Oudovenko, "On the theory of superconductivity in the extended Hubbard model. Spin-fluctuation pairing", Eur. Phys. J. B, 86 (2013), 115, 15 pp.

[39] L.F. Feiner, J.H. Jefferson, R. Raimondi, "Effective single-band models for the high- $T_{\mathrm{c}}$ cuprates. I. Coulomb interactions", Phys. Rev. B, 53:13 (1996), 8751-8773. 